\title{
CONTRIBUCIÓN AL ESTUDIO DE LAS MIELES DE EXTREMADURA (ESPAÑA)
}

\author{
María C.TELLERIA y Juan A. DEVESA
}

RESUMEN. Contribución al estudio de las mieles de Extremadura (España). Se analizaron 39 muestras de miel procedentes de Extremadura (España), en las que se identificaron 90 tipos morfológicos. 18 muestras fueron mixtas y las restantes monoflorales, producidas a expensas de: Eucalyptus camaldulensis, Lavandula stoechas s.l., Rosmarinus officinalis, Echium plantagineum y Retama sp.-Cytisus $\mathrm{sp}$. El análisis cuantitativo puso de manifiesto que la mayoría de las muestras contenían entre 100 000-500 000 granos de polen en 10 $\mathrm{g}$ de miel. Se discute la elevada representatividad de las plantas poliníferas.

Palabras clave. Miel, análisis polínico, Melisopalinología, Extremadura, España.

ABSTRACT. A contribution to the knowledge of honeys from Extremadura (Spain). 39 honey samples from the region of Extremadura (Spain) were analyzed, in which 90 morphological types of pollen were recognized. Eighteen honey samples showed a mixed floral origin while the remaining came from Eucalyptus camaldulensis, Lavandula stoechas s.l., Rosmarinus officinalis, Echium plantagineum and Retama sp.-Cytisus sp. type. The quantitative analysis showed that most of the samples contained from 100.000 to 500.000 pollen grains per $10 \mathrm{~g}$. of honey. The high level of pollen in the samples from polliniferous plants is discussed.

Key words. Honey, palynological analysis, Melissopalynology Extremadura, Spain.

\section{INTRODUCCIÓN}

La apicultura constituye en España una práctica muy importante y que ha visto en las últimas décadas un notable resurgir (Devesa et al., 1987), especialmente en aquellas regiones que gozan de una flora melífera de excelencia y en las que las actividades apícolas aportan beneficios económicos a sumar a los escasos recursos naturales existentes. En particular, en la región extremeña las prácticas apícolas constituyen una actividad muy arraigada (Frades, 1987), haciendo que esta Comunidad ocupe con sus $3.488 .000 \mathrm{~kg}$ (en 1991) la segunda posición en cuanto a producción de miel en el contexto nacional, después de la

Este trabajo fue realizado con motivo de una estancia de 6 meses de duración de uno de los autores (M.C. Tellería) en la Universidad de Extremadura, disfrutando de una beca del Programa «Estancias Temporales de Científicos y Tecnólogos Extranjeros en España» concedida por el Ministerio de Educación y Ciencia. 
Comunidad Valenciana, y el primer puesto en cuanto a producción de polen $(250.000$ el mismo año; Devesa, 1992). La apicultura en Extremadura adquiere su mayor importancia en aquellos enclaves más abruptos o con suelos de poca calidad y, por tanto, no explotados agrícolamente, donde la vegetación mediterránea aparece en distintas etapas de degradación y/o recuperación (Devesa, 1992). Las áreas más importantes se localizan en las serranías de Los Ibores-Villuercas-Siberia, Gata-Las Hurdes y San Pedro, donde se concentran grandes masas de vegetación arbustiva (v.g. jarales, brezales y cantuesales) y donde se ubican el mayor número de colmenas (Torres 1987; Del Solar, com.pers.).

Sin embargo, hasta la fecha no son muchos los estudios sobre mieles extremeñas, pudiéndose destacar las contribuciones de Gomez y Sáenz (1980), Montero y Muñoz (1990) y Montero y Tormo (1990a y b), en su mayoría centradas en alguna comarca en particular. Por ello, con el presente trabajo se pretende paliar en cierta medida esta situación, al efectuarse un estudio melitopalinológico de muestras procedentes de la mayor parte del territorio.

\section{MATERIAL Y MÉTODOS}

Se analizaron 39 muestras de miel correspondientes a la cosecha 1991, que fueron suministradas por las Cooperativas de Apicultores y cuya procedencia se indica en las siguientes localidades.

BADAJOZ: 1- Fregenal de la Sierra. 2- Monte la Real. 3- Herrera del Duque. 4- Monterrubio de la Serena. 5- Manchita. 6- Sancti-Spiritu. 7- Medina de las Torres. 8- La Roca de la Sierra. 9- Guareña. 10- Burguillos del Cerro. 11- Fuenlabrada. 12Valverde de Burguillos. 13- Palomas. 14- Zalamea la Real. 15- Fuente del Arco. 16- Manchita. 17Navalvillar de Pela. 18- Olivenza. 19- Talavera la Real. 20- Guadajira. 21- Mirandilla.

CÁCERES: 22- Logrosán. 23- Cañamero. 24Cañamero. 25-Valverde del Fresno. 26-Acehuche.
27- Plasencia. 28- Zarza la Mayor. 29- Puerto Perales. 30- Sierra de Ovejuela. 31- Valverde del Fresno. 32- Peraleda de San Román. 33- Morcillo. 34- Castañar de Ibor. 35- Valdecasa del Tajo. 36 Villar del Pedroso. 37- Santa Marta de Magasca. 38- Logrosán. 39- San Telmo.

Tanto para el análisis cualitativo como para el cuantitativo, se siguieron las técnicas propuestas por Louveaux et al. (1978). La determinación de los tipos morfológicos se hizo sobre la base de la Palinoteca existente en la Unidad de Botánica del Departamento de Biología y Producción de los Vegetales de la Universidad de Extremadura, así como con la ayuda del Atlas Polínico de Andalucía Occidental (Valdés et al., 1987). Para establecer los porcentajes de cada tipo morfológico, fueron contados 400 granos de polen por muestra (Montero y Tormo, 1990a).

Se siguieron distintos criterios para la tipificación de mieles monoflorales: Serra y Cañas (1988) para las de Eucalyptus sp., Serra et al. (1987) para las de Lavandula y Rosmarinus, y Sala (1989) para las de Echium plantagineum.

\section{RESULTADOS}

Se determinaron 90 tipos morfológicos de los cuales 48 corresponden al nivel genérico (incluyendo 7 tipos), 37 al nivel específico, y 5 a familia (tab. 1). El número de tipos por muestra osciló entre 10 y 32. La figura 1 exhibe la frecuencia de aparición de los taxones representados en las muestras de miel.

Los tipos morfológicos determinados corresponden a diversas familias: Fabaceae (15 tipos); Asteraceae (12 tipos); Apiaceae (6); Liliaceae (5); Cistaceae (4); Lamiaceae, Ericaceae, Ranunculaceae, Rosaceae, y Scrophulariaceae (3 tipos); Boraginaceae, Fagaceae, Oleaceae, Poaceae y Resedaceae con 2 tipos y finalmente con un sólo tipo: Amaranthaceae, Anacardiaceae, Aquifoliaceae, 
Brassicaceae, Campanulaceae, Caryophyllaceae, Cucurbitaceae, Cyperaceae, Euphorbiaceae, Fumariaceae, Lythraceae, Myrtaceae, Papaveraceae, Plantaginaceae, Polygonaceae, Rafflessiaceae, Rhamnaceae, Salicaceae, Simaroubaceae, Solanaceae, Ulmaceae, Urticaceae y Vitaceae (tab. 1).

Del total de muestras analizadas 18 fueron milflores y el resto monoflorales, estas últimas elaboradas a partir de: Eucalyptus camaldulensis (11 muestras), Echium plantagineum (3 muestras), Lavandula stoechas s.1. (4 muestras), Rosmarinus officinalis (2 muestras) y Retama sphaerocarpa (1 muestra). Ninguna de las muestras analizadas resultó ser de mela, si bien éstas se producen en la región a expensas de los exudados de las bellotas de encina (Quercus rotundifolia; (Devesa 1992).

En cuanto al análisis cuantitativo, 8 muestras pertenecen al grupo II (entre $20000 \mathrm{y}$ 100000 granos/10 g de miel), 23 al grupo III (entre 100000-500000 granos/10 g), 4 al grupo IV (entre 500000-1000000 granos $/ 10 \mathrm{~g}$ ) y $4 \mathrm{al}$ grupo V (más de 1000000 granos/10 g).

\section{DISCUSIÓN}

Origen botánico: una característica bastante general y llamativa de las mieles de Extremadura es la elevada representatividad de los taxones poliníferos, pues en algunas muestras sus granos alcanzan la categoría de polen secundario tal como sucede con: Phillyrea sp.,Papaver sp. y Quercus sp. (tab. 1). Probablemente La utilización de la colmena tipo Layens, que ha reemplazado en su mayor parte a los tradicionales «corchos», es la responsable de esta particularidad, ya que en el interior de la misma la miel y el polen comparten los mismos cuadros; la castración de estos cuadros propicia que el contenido de las celdillas polínicas «contamine» la miel, de manera similar a lo que sucede en las mieles obtenidas por prensado. En apoyo a esta suposición abogan los resultados del análisis cuantitativo, ya que una parte importante de las muestras están comprendidas entre las clases IV y V, que corresponden a mieles enriquecidas con polen durante el proceso de extracción (Louveaux et al., 1978). Estas observaciones también concuerdan con los resultados obtenidos por Fernandez y Ortíz (1994)

Merece resaltarse que las denominaciones asignadas a las mieles por los apicultores en el territorio, a veces no coincidieron con los resultados de los análisis polínicos. Así, por ejemplo, en la miel suministrada originariamente como «de castaño», el porcentaje de polen de esta planta nunca alcanzó el $90 \%$ requerido (Louveaux et al., 1978), y lo mismo sucedió con las de «brezo», donde el polen de Erica spp. nunca llegó a superar el $50 \%$ del total requerido (Aira et al. 1990).

Origen Geográfico: según la combinación de tipos dominantes y secundarios (Maurizio, 1971), las mieles extremeñas se caracterizan por presentar en general una combinación de pólenes procedentes tanto de taxones nectaríferos, sobre todo Eucalyptus camaldulensis, Lavandula stoechas, Rosmarinus officinalis, Echium plantagineum, Helianthus annuus, Campanula-Jasione, Rubus ulmifolius, Brassicaceae, Retama-Cytisus y Erica sp, como de los exclusivamente poliníferos, como Phillyrea spp., Quercus suber, Quercus rotundifolia, Olea europaea y Papaver sp., entre otros. La mayoría de estos taxones presentan, además, una elevada frecuencia de aparición, tal como se observa en el espectro polínico (fig.1).

La caracterización de las mieles por comarcas geográficas dentro del territorio es difícil de realizar, debido sobre todo a la transhumancia que se practica en la región. Esta particularidad se ve reflejada en las mieles, donde es posible hallar polen de plantas melíferas de distinta fenología e incluso pertenecientes a cultivos poco importantes en 


\section{MUESTRAS}

TAXONES

AMARANTHACEAE

Amaranthus sp.

ANACARDIACEAE

Pistacia sp.

APIACEAE

Apiaceae s.l.

Conium sp.-Amni sp.

Daucus sp.

Eryngium sp.

T. smimium sp.

Torilis sp.

AQUIFOLIACEAE

Ilex aquifolium

ASTERACEAE

T. Aster sp.

T. Bellis annua

Carduus sp.

Calendula sp.

Carlina sp.

Centaurea sp.

Cichorium sp.

Cynara humilis

Helianthus annus

T. Picris sp.

Scolynus hispanicus

Xanthium sp.

BORAGINACEAE

Echium plantagineum

Myosotis sp.

BRASICACEAE

$T$. Raphanus raphanistrum

CAMPANULACEAE

Campanula sp.-Jasione sp.

CARYOPHYLLACEAE s.l.

CISTACEAE

Cistus crispus-C. albidus

C. ladanifer

C. monspeliensis-C. salvifolius

Halimium sp.-Tuberaria sp.

CUCURBITACEAE

Cucumis myriocarpus

CYPERACEAE

EUPHORBIACEAE

Securinega tintorea

ERICACEAE

Calluna vulgaris

Erica aborea

E. australis-E. umbellata

FABACEAE

Anthyllis lotoides

Coronilla sp.

Lathyrus sp.

Lotus sp.

Lupinus sp.

Medicago sp.

Melilotus sp.

Melilotus messanensis

Ononis sp.

T. Retama sp.-Cytisus sp.

Trifolium arvense

T. pratense

T. repens

Ulex eriocladus

Vicia sp.

FAGACEAE

Castanea sativa

Quercus rotundifolia-Q. sube FUMARIACEAE

Hypecoum sp.

LAMIACEAE

Lavandula stoechas

Mentha sp.

Salvia sp.-Rosmarinus sp.
1233456677899101112131415161718192021222324252627282930313233343536373839

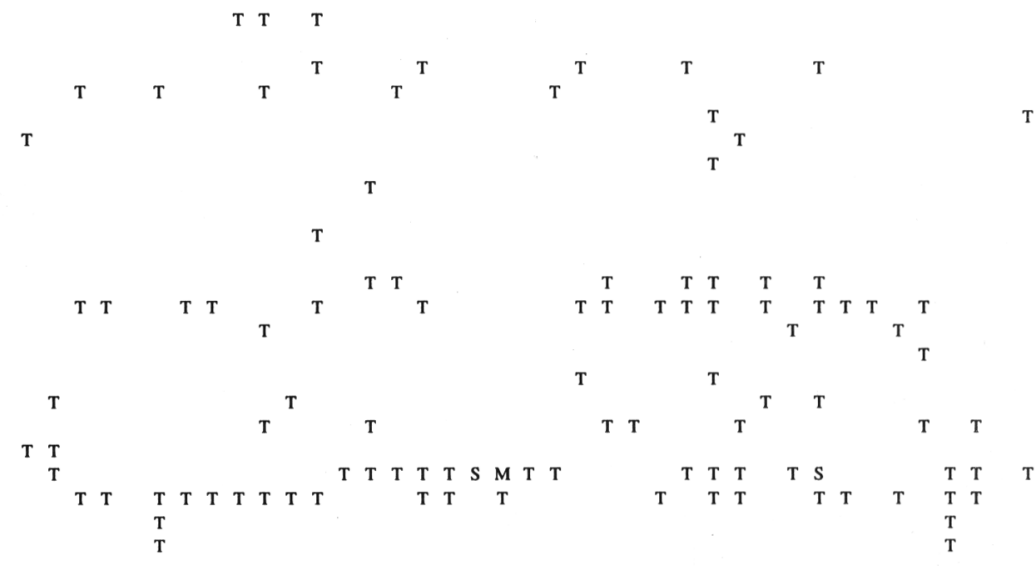

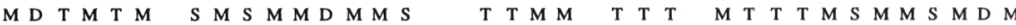
$\mathrm{T}$

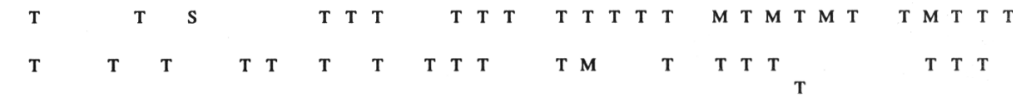

$\begin{array}{lllllllllll}T & T & T & T & T & T & M & T & T & T\end{array}$

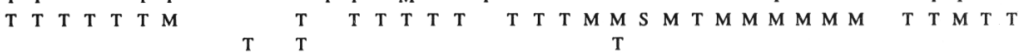
$\begin{array}{lllllll}T & T & T & T & T & T & T\end{array}$ $\mathbf{T}$

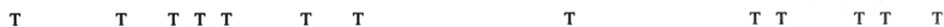

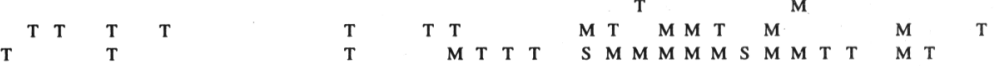
$\mathbf{T}$ $\begin{array}{lll}T & & T \\ T & T & T \\ & & T\end{array}$

T $\quad \mathbf{T}$

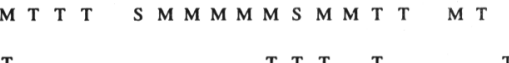

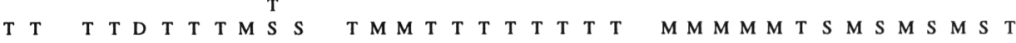

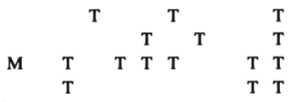
$\mathbf{T}$

$\begin{array}{llllll}T & S & T & S & T & S\end{array}$

$\begin{array}{llll}T & T & S & T\end{array}$

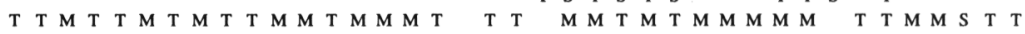
$\mathrm{T}$ T $\quad \mathrm{T}$

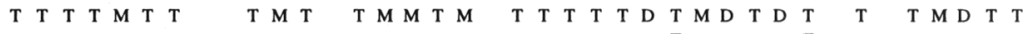
D 


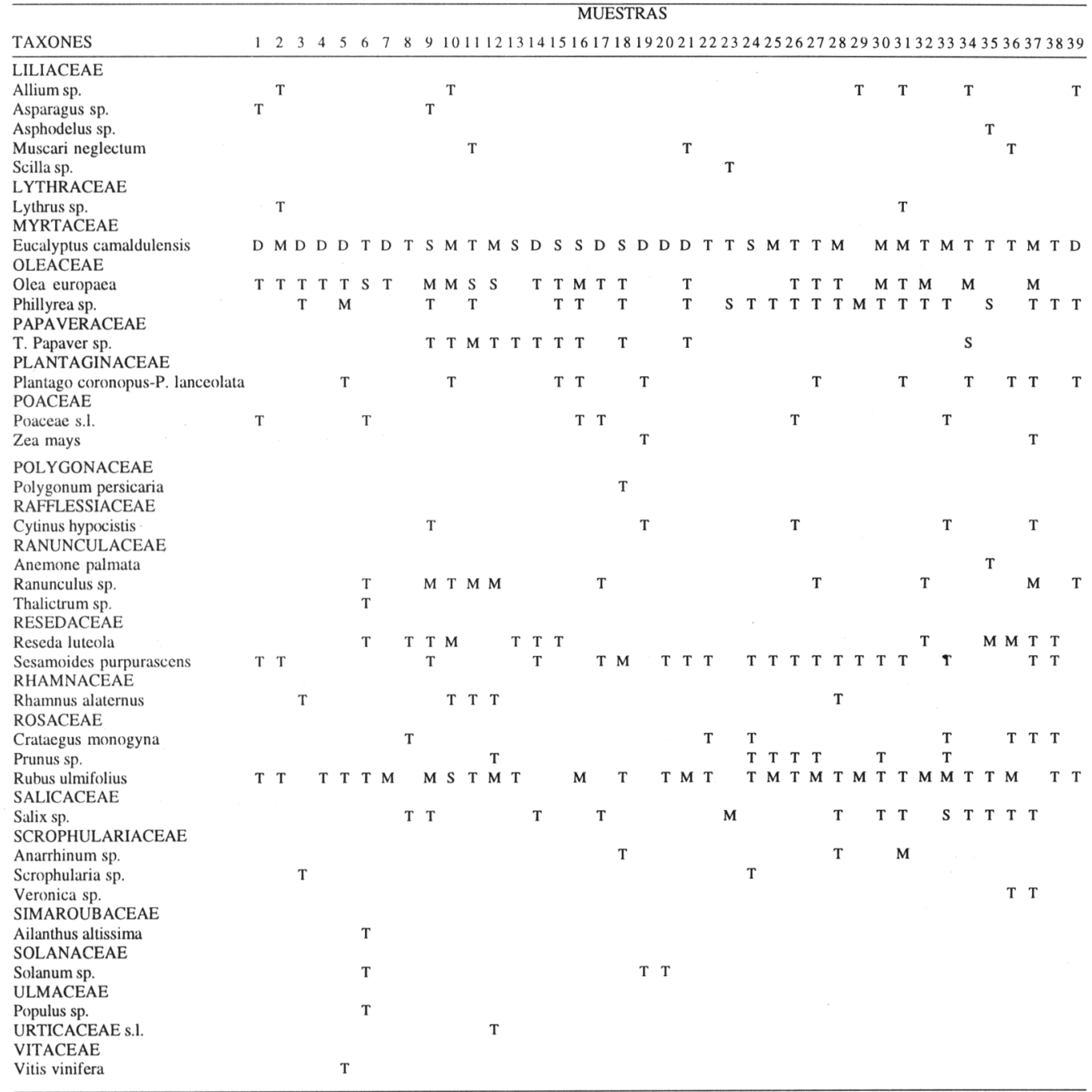

Tabla 1. Abundancia de los tipos morfológicos determinados en la mieles. D: polen dominante. S: polen secundario. M: polen de menor importancia. T: polen en traza. Abundance of pollen-types in the honeys samples of Extremadura according with Maurizio's classification (1971).

el área de procedencia de algunas mieles, como sucede en las muestras procedentes de Valverde del Fresno, donde el girasol aparece en un porcentaje elevado (tab. 1, muestra 31). No obstante, las mieles «enriquecidas» en polen de castaño (tab. 1, muestra 22) son particularmente frecuentes en Ibores-Villuercas (vide Montero y Tormo 1990b) y en el extremo más septentrional del territorio, sobre todo por la ubicación en estas zonas de las mayores masas de castañares, e igual sucede con las, enriquecidas en brezo, particularmente abundantes en Gata-Las Hurdes por igual motivo. 


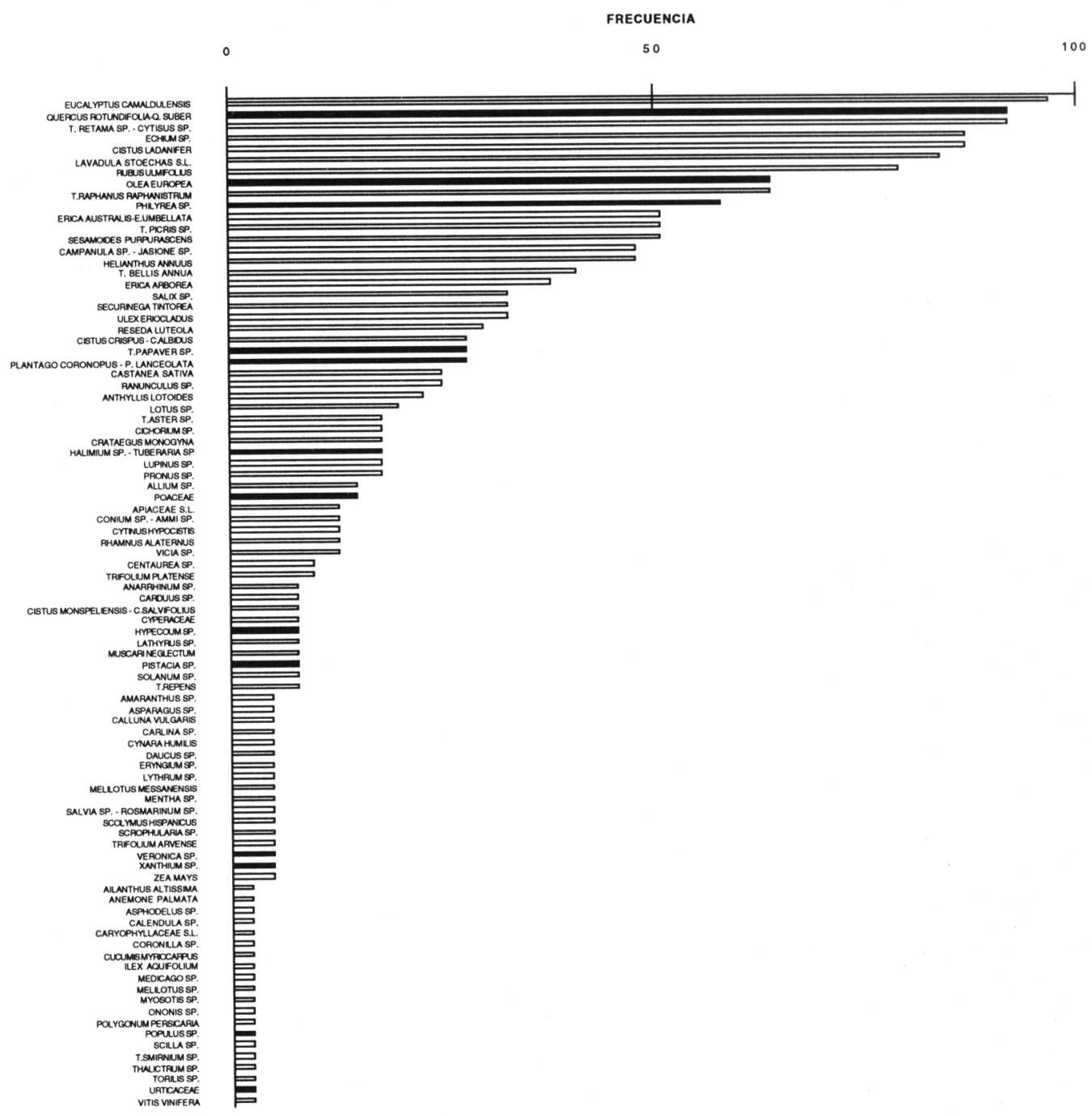

Figura 1. Frecuencia de aparición de los tipos polínicos identificados en las mieles de Extremadura. Resaltados en negrita las barras de los taxones poliníferos. Frequence of pollen-types in the honey samples of Extremadure.

\section{CONCLUSIONES}

A pesar de que la colmena de tipo Layens está muy difundida en la zona por su facilidad de manejo y transporte (Viladomat 1982), los análisis palinológicos ponen de manifiesto que dicha elección, unida a la práctica transhumante, limitan las interpretaciones del espectro polínico, tanto para determinar el origen botánico de las mieles como su origen geográfico. Estas limitaciones deberían ser consideradas, sobre todo si se pretende tipificar 
las mieles para la comercialización. No obstante, en aquellos enclaves donde las características botánicas posibilitan la producción de mieles monoflorales, como las de brezo o castaño (que no alcanzaron el porcentaje de polen mínimo requerido en las muestras estudiadas), deberían analizarse las condiciones de producción en particular, al objeto de poder garantizar la producción de miel monofloral, en muchos casos más apreciada y rentable desde el punto de vista económico.

\section{BIBLIOGRAFÍA}

AIRA-RODRÍGUEZ, M.J., P. RAMIL-REGO y M.P. SAA-MONTERO -1990-Identificación polínica de Ericaceae en mieles gallegas. Acta Bot. Malacitana, 15: 27-32.

DEVESA, J.A. -1992- Flora apícola, su interés y productos derivados: miel y polen. Actas de las Jornadas Técnicas sobre Obtención de Productos Ganaderos Naturales en el Ecosistema de la Dehesa, 1: 151-161. Zafra, Badajoz.

DEVESA, J.A., P. ORTIZ y A. MUÑOZ -1987Breve reseña histórica de la apicultura en España. Vida Apícola, 25: 63-65.

FERNÁNDEZ, I. y P.L. ORTIZ -1994- Pollen contamination of honey by bees inside the hive. Grana, 33: 282-285.

FRADES, D. -1987- La apicultura extremeña Apuntes para su historia, 288 pp. S.E.Y.C.A. Badajoz.

GÓMEZ FERRERAS C. y C. SÁENZ DE RIVAS 1980- Análisis polínico de las mieles de Cáceres. Anales Jard. Bot. Madrid, 36: 191-201.

LOUVEAUX J., A. MAURIZIO y G. VORWHOL. -1978- Methods of Melissopalynology.Bee World , 59 (4): 139-157.

MAURIZIO, A. -1971- Le spectre pollinique des miels luxembourgeois. Apidologie, 2 (3): 221 238.

MONTERO, I. y A. MUÑOZ -1990-Análisis de mieles de la Sierra de Villuercas-Ibores (Cáceres, España). Actas VII Simposio de Palinología (A.P.L.E.), 397-403. Granada.
MONTERO, I. y R. TORMO -1990a- Análisis polínico de mieles de cuatro zonas de montaña de Extremadura. Anales A.P.L.E., 5: 71-78.

MONTERO, I. y R. TORMO -1990b- Discriminación del origen geográfico de mieles de Extremadura por métodos estadísticos. Actas VIII Simp. Palinol. 397-403. Puerto de Santa Cruz (Tenerife, España).

SALA LLINARES, A. -1989- Mieles del Mediterráneo español. Vida Apícola , 37: 4751.

SERRA, B.J., A. GÓMEZ PAJUELO y F. GONELL GALINDO -1987-Composición, propiedades físico-químicas y espectro polínico de algunas mieles monoflorales españolas. Alimentaria, XXIV (185): 61-84.

SERRA, B.J. y S. CAÑAS LLORIA -1988Caratteristiche fisico-chimiche, composizione e spettro pollinico del miele di Eucalipto (Eucalyptus spp.) prodotto in Spagna. Apicoltura, 4: 59-81.

TORRES NAVARRO, J. -1987-Censo apícola de Extremadura, 1986., 12 pp. S.E.Y.C.A. Badajoz. VALDÉS, B., J. DÍEZ e I. FERNÁNDEZ -1987Atlas polínico de Andalucía Occidental pp. 450. Instituto de Desarrollo Regional, Universidad de Sevilla, Excma. Diputación de Cádiz. Utrera (Sevilla).

VILADOMAT, J. -1982- La colmena Layens. Vida Apícola , 4:21-22.

Aceptado para su publicación en Mayo de 1995

Dirección de los autores:M.C. Tellería: Cátedra de Palinología, Facultad de Ciencias Naturales, Paseo del Bosque s/n, 1900 La Plata, República Argentina. J.A. Devesa: Departamento de Biología y Producción de los Vegetales: Unidad de Botánica, Avenida de Elvas s/n, 06071-Badajcz, España. 